\title{
Catching the therapeutic window of opportunity in early initial-onset Vogt-Koyanagi-Harada uveitis can cure the disease
}

\author{
Carl P. Herbort Jr. • Ahmed M. Abu El Asrar • Masuru Takeuchi • \\ Carlos E. Pavésio - Cristobal Couto - Alireza Hedayatfar - Kazuichi Maruyama • \\ Xi Rao $\cdot$ Sukhum Silpa-archa $\cdot$ Thanapong Somkijrungroj
}

Received: 22 April 2018/Accepted: 4 June 2018/Published online: 11 June 2018

(C) The Author(s) 2018

\begin{abstract}
Purpose Vogt-Koyanagi-Harada (VKH) disease is a primary autoimmune granulomatous choroiditis that begins in the choroidal stroma. The aim of this review was to gather a body of evidence for the concept of a window of therapeutic opportunity, defined as a time interval following initial-onset disease during which adequate treatment will substantially modify the disease outcome and possibly even lead to cure,
\end{abstract}

\section{P. Herbort Jr. ( $₫)$}

Retinal and Inflammatory Eye Diseases, Centre for Ophthalmic Specialized Care (COS), Clinic Montchoisi Teaching Centre, Rue Charles-Monnard 6,

1003 Lausanne, Switzerland

e-mail: cph@herbortuveitis.ch

\section{P. Herbort Jr.}

Department of Ophthalmology, University of Lausanne, Lausanne, Switzerland

\section{A. M. Abu El Asrar ( $\square)$}

Department of Ophthalmology and Dr. Nasser Al-Rashid Research Chair in Ophthalmology, College of Medicine, King Saud University, Riyadh, Saudi Arabia e-mail: abuelasrar@yahoo.com

\section{Takeuchi}

Department of Ophthalmology, National Defence Medical College, Tokorozawa, Saitama, Japan similar to what has been described for rheumatoid arthritis.

Methods We reviewed the literature and consulted leading experts in $\mathrm{VKH}$ disease to determine the consensus for the notion of a therapeutic window of opportunity in VKH disease.

Results We found a substantial body of evidence in the literature that a therapeutic window of opportunity exists for initial-onset acute uveitis associated with VKH disease. The disease outcome can be

\section{E. Pavésio}

National Institute for Health Research, Biomedical

Research Centre at Moorfields Eye Hospital, NHS

Foundation Trust and UCL, Institute of Ophthalmology,

London, UK

C. Couto

Department of Ophthalmology, University of Buenos

Aires, Buenos Aires, Argentina

A. Hedayatfar

Noor Eye Hospital and Rassoul Akram Hospital, Iran

University of Medical Sciences, Tehran, Iran

K. Maruyama

Department of Innovative Visual Science, Graduate

School of Medicine, Osaka University, Osaka, Japan

X. Rao

Department of Ophthalmology, General Police Hospital, University of Chile, Santiago, Chile 
substantially improved if dual systemic steroidal and non-steroidal immunosuppressants are given within 2-3 weeks of the onset of initial VKH disease, avoiding evolution to chronic disease and development of "sunset glow fundus." Several studies additionally report series in which the disease could be cured, using such an approach.

Conclusions There is substantial evidence for a therapeutic window of opportunity in initial-onset acute VKH disease. Timely and adequate treatment led to substantial improvement of disease outcome and prevented chronic evolution and "sunset glow fundus," and very early treatment led to the cure after discontinuation of therapy in several series, likely due to the fact that the choroid is the sole origin of inflammation in VKH disease.

Keywords Vogt-Koyanagi-Harada disease ·

Therapeutic window of opportunity stromal choroiditis · Autoimmune disease $\cdot$ Indocyanine green angiography $\cdot$ Immunosuppressive therapy $\cdot$ Sunset glow fundus

A window of opportunity is a brief time interval during which a suitable action will achieve success. Once this period passes, the window is closed and the desired outcome is no longer achievable. The therapeutic window of opportunity is a well-established concept of critical importance in medicine, specifically for rheumatoid arthritis (RA), and considerable attention has been paid to its timing [1]. In a case-control study, Nell et al. [2] showed that there was a window of opportunity for highly successful RA treatment in the first year, especially within the first 3 months of therapy, and they suggested that early diagnosis and therapy is a crucial step toward achieving optimal control of RA progression and prognosis. This window represents a very early disease phase in which therapeutic disease modification is more successful,

\footnotetext{
S. Silpa-archa

Department of Ophthalmology, Faculty of Medicine, Rajavithi Hospital, College of Medicine, Rangsit University, Bangkok, Thailand

T. Somkijrungroj

Department of Ophthalmology, Faculty of Medicine,

Chulalongkorn University, Bangkok, Thailand
}

likely because the underlying disease process is not fully matured. A growing body of evidence has emphasized the consistent clinical and radiographic benefits of prompt initiation of disease-modifying anti-rheumatic drugs (DMARDs) for patients with rheumatoid arthritis during the early stages of the disease. Therapeutic intervention during this period alters disease progression in such a way that chronicity is reduced, resulting in a greater proportion of patients in drug-free remission after treatment withdrawal as well as reduced mortality. These findings have led to changes in rheumatoid arthritis treatment paradigms, with an increasing emphasis on early diagnosis and treatment $[3,4]$.

Timely and appropriate therapeutic intervention may substantially modify the outcome of ocular inflammatory disorders in a similar way.

This may have important consequences for the care of initial-onset acute uveitis associated with VogtKoyanagi-Harada (VKH) disease, as delivering the right therapies at the right time could reduce the burden of the disease and substantially alter the outcome. VKH pathogenesis in the eye is characterized by an autoimmune reaction against stromal melanocytes or associated proteins [5-8]. It is considered a primary stromal choroiditis because the inflammatory reaction electively starts within the choroidal stroma [9]. The initial-onset acute disease typically exhibits granulomatous choroiditis with exudative retinal detachment and optic disk hyperemia and swelling, followed by anterior segment involvement and eventual development into chronic recurrent granulomatous anterior uveitis if not properly treated, with typical "sunset glow fundus" (SGF) and chorioretinal atrophy [10-13].

Ocular involvement is limited to subclinical occult choroiditis during the prodromal phase of initial-onset disease. Acute clinically apparent ocular disease develops after choroiditis spills over into the neighboring structures. This early stage of disease represents the therapeutic window of opportunity, during which the disease is amenable to appropriate therapeutic intervention. If the window closes without sufficient treatment, evolution will irremediably progress toward chronic disease (smoldering or recurrent) which is much more refractory to treatment and needs prolonged and repeated therapeutic intervention [12].

Chronic disease resulting from improper treatment must be distinguished from initial-onset disease which 
may be substantially impacted by treatment and susceptible to long-term remission and even cure. Intraocular inflammation in insufficiently treated VKH disease will proceed to recurrent granulomatous anterior and posterior uveitis and typical sunset glow fundus (SGF), even when systemic monotherapy is given early in disease [10-16].

Therefore, we must consider both the time interval for the window of opportunity, and what is appropriate therapy for the treatment of initial-onset VKH disease.

The exact timing of the therapeutic window is difficult to determine, but it is clear that the earlier appropriate therapy begins, the higher the chances to achieve an optimal outcome. One study defined the therapeutic window as 2 weeks following disease onset [17]. Although each patient is different, it is accurate to conclude that the window of therapeutic opportunity closes approximately 2-3 weeks after initial onset [17]. Immediate use of non-steroidal immunosuppression has the advantage to result in immediate inflammation suppressive activity if immediately acting agents such as cyclosporine are used. If agents with delayed activity are used, such as mycophenolic acid, it results in efficient activity at the needed time when corticosteroid tapering has started.

Fortunately, much more precise information is available about the appropriate therapy for initialonset VKH disease.

\section{Corticosteroid monotherapy has been shown to be inappropriate even if given within an adequate timeframe}

Convincing evidence has become available so far to be able to say that systemic corticosteroid monotherapy is not sufficient even if given at an early stage of disease to prevent chronic evolution. A recent publication reported a high percentage of clinical recurrence in VKH patients given early high-dose corticosteroids with slow taper, as $79 \%$ of patients progressed to chronic recurrent disease and 38\% developed subretinal fibrosis [18]. Additional studies by Chee et al. [19], and Keino et al. [20], showed that steroid monotherapy given at initial-onset disease was incapable of preventing chronic evolution, which is significantly associated with more severe anterior segment inflammation, has a worse visual acuity [12], a worse mean retinal sensitivity [21] and more frequent complications compared with initial-onset acute VKH disease receiving appropriate therapy $[11,12,21]$. Moreover, it was shown that chronic recurrent disease was far more refractory to treatment [21].

The result of chronic ongoing inflammation of the choroid is SGF, which indicates insufficient therapy. Keino et al. [22] followed 102 patients with VKH disease from initial onset who were treated with highdose corticosteroid therapy. SGF developed in $67.6 \%$ of patients, and the mean duration before SGF appearance was $4.2 \pm 2.7$ months. Lai et al. [23] similarly demonstrated SGF development in $51.4 \%$ of patients who received oral corticosteroids during their first attack of VKH disease. These data represent substantial evidence indicating that systemic corticosteroid monotherapy cannot control progressive subclinical choroidal inflammation in most cases, even when given shortly after onset of the disease.

\section{Combination of steroidal and non-steroidal immunosuppression is required to treat initial- onset acute VKH disease in order to prevent chronic evolution}

Because numerous studies have shown that corticosteroid monotherapy is insufficient to prevent chronic evolution, combinations of corticosteroids with firstline non-steroidal immunosuppressive therapies such as cyclosporine, azathioprine, mycophenolate mofetil, anti-tumor necrosis factor- $\alpha$ agents, or others have been proposed to achieve better control of the uveitis, facilitate earlier tapering of corticosteroids, and avoid chronic evolution in acute initial-onset disease [17, 24-27]. First-line immunosuppression is defined here as the initiation of immunosuppression at the onset of disease concomitant to corticosteroids and not thereafter, as is the case in most studies speaking of first-line immunosuppressive therapy.

Subclinical choroiditis is the reason for chronic evolution and is clearly identified by ICGA, uncovering active choroidal inflammation in an apparently quiescent eye during follow-up while monitoring corticosteroid tapering [28-33]. This shows that the standard corticosteroid monotherapy is indeed suppressing clinically apparent disease, but is insufficient to suppress choroidal inflammation [32]. Subclinical evolution of choroidal inflammation has also been 
clearly documented by several groups investigating smoldering disease with the help of ICGA [28-33], explaining that the development of SGF despite corticosteroid therapy results from the progressive loss of stromal melanocytes due to the ongoing insufficiently controlled immunological process $[19,32]$. This is a strong incentive to maintain sufficiently dosed therapy including first-line nonsteroidal immunosuppressive drugs, even in the subacute phase and during the corticosteroid tapering process, to be sure to eradicate choroidal inflammation [24-33]. An Indian group proposed early and highdose triple agent immunosuppression in 2006 and reported favorable outcomes with disease remission in all five reported patients [34].

\section{Association of steroidal and non-steroidal immunosuppression induces long-term remission and even cure of VKH disease}

Abu El-Asrar et al. [24] determined the effectiveness of mycophenolate mofetil (Cellcept ${ }^{\circledR}$ ) combined with systemic corticosteroids as first-line therapy for very early initial-onset acute VKH disease without AC inflammatory signs [26]. They compared the outcomes in this group with those of a group of patients with acute VKH uveitis treated with corticosteroid monotherapy or with the previously reported delayed addition of immunomodulatory therapy [35]. The use of Cellcept ${ }^{\circledR}$ as first-line therapy combined with systemic corticosteroids was safe and effective in the treatment of acute uveitis associated with VKH disease, and significantly reduced the development of chronic recurrent inflammation and late complications and also improved visual outcome. None of the patients developed SGF, suggesting that it was also effective in controlling progressive subclinical choroidal inflammation. Mycophenolate mofetil was also effective in preventing the development of vitiligo, poliosis, alopecia, and sensory hearing loss [24, 26]. In a series [26] of 38 patients with initial-onset acute uveitis associated with VKH disease, 22 (57.9\%) patients were able to discontinue treatment without relapse of inflammation. The median durations of corticosteroid therapy and mycophenolate mofetil therapy in these patients were 17.5 and 20.2 months, respectively, and the patients were off treatment for a median period of 25.5 months.
Another study compared medium-dose corticosteroid monotherapy to high-dose corticosteroid therapy combined with non-steroidal immunosuppression and showed that all patients in the corticosteroid monotherapy group showed SGF after 4 months. ICGA also showed the persistence of numerous hypofluorescent dark dots (HDDs) in the corticosteroid group, while no patient showed SGF nor HDDs in the combined group [32].

In a series of $9 \mathrm{VKH}$ patients receiving combined steroidal and non-steroidal immunosuppression with ICGA assisted monitoring of therapy to detect subclinical recurrence of choroiditis, a high proportion $(7 / 9,78 \%)$ of cases "healed" with no recurrent activity within a mean follow-up therapy-free period of $26 \pm 14.8$ months, and a low proportion of cases developed SGF $(3 / 9,33 \%)$ [29]. However, the mean duration of treatment was 27.3 months \pm 38.2 months (range 9-114), much longer than the 6-9 months typically recommended in the literature [29]. These results indicate that early, combined, sustained, and if possible ICGA-monitored treatment is able to modify the VKH disease phenotype, avoiding SGF and achieving cure, much in the same fashion as early and sustained therapy changes the phenotype of birdshot retinochoroiditis [36]. Treatment duration is variable and individually different, depending on the time-point of treatment initiation, disease severity, and individual susceptibility to treatment, which may include biomarkers indicating therapy response in the future [37]. Treatment should not be stopped if subclinical ICGA-detected choroiditis remains present, and the duration should be 15 months at minimum, and ideally closer to 24 months [29].

\section{Initial-onset acute VKH, a condition particularly prone to benefit from the concept of therapeutic window of opportunity}

The particularity of initial-onset acute VKH is that inflammation develops exclusively within the choroid, meaning that intraocular inflammation completely subsides once choroiditis is completely under control, as there is no other origin of inflammation. However, we know by now that all choroidal inflammation may not be silenced even when clinically apparent disease appears to be under control. ICGA monitoring of VKH 
patients under treatment has shown that choroidal inflammation is still present in most cases once clinical signs as well as morphological and functional parameters have normalized [28-33, 38-40], and the persistence of this choroidal subclinical disease explains the evolution toward SGF in most insufficiently treated cases [20, 22, 41].

Zero tolerance to choroiditis must therefore be the aim if cure of the disease is to be achieved. Some authors proposed ICGA monitoring for regression of choroidal disease in the convalescent phase of disease and long-term tapering of therapy until absence of recurrence of choroidal subclinical disease, i.e., absence of recurrence of hypofluorescent dark dots (HDDs) on ICGA [29]. Ideally, if possible and available to the clinician, meaningful ICGA monitoring presupposes an ICGA every 5-6 weeks during the first 4-6 months and then every 2-3 months during the long-term tapering period [29]. Performing an ICGA every 6 months in the follow-up of VKH disease does not allow for timely treatment modification, but is nevertheless useful in order to provide information about the presence or absence of subclinical occult choroiditis [42]. ICGA is very reactive and precise for evaluating disease activity in the choroid and allows for therapy readjustment and safe tapering [43]. The treatment durations reported in textbooks or in published literature are not long enough to achieve zero tolerance of choroiditis and safe withdrawal of treatment [29, 44]. With optimal management including early combined steroidal and first-line nonsteroidal immunosuppression, SGF should not be considered part of the natural course of the disease, but rather the result of insufficient treatment that failed to stop subclinical choroidal inflammation [45]. In contrast to many reports, a recent report stated that corticosteroid therapy in initial-onset disease was sufficient to achieve a satisfactory outcome with nonsteroidal immunosuppression necessary in only $15.3 \%$ of cases [46]. No information was given, however, on the rate of clinical chronic evolution, nor on occult chronic evolution as ICGA was not performed. No information was given either on treatment-free followup. Sunset glow fundus was reported at the rate of close to $50 \%$, anterior and posterior recurrence at the rate of $22.5 \%$ and complications at the rate of $21.2 \%$. Judging by these numbers, chronic evolution must not have been negligible.

\section{Perspective: the therapeutic window of opportunity in VKH}

In summary, VKH disease is a primary stromal choroiditis with inflammation initiating exclusively from the choroid, the structure that should be targeted by therapy. The choroidal compartment is easily accessible to systemic therapy, unlike the retina. There is increasing evidence that a substantial proportion of cases can be cured before depigmentation occurs if treatment is conducted with a zero tolerance for recurrence of the mostly subclinical choroiditis that can be detected by ICGA, Three crucial conditions must be satisfied to achieve this goal: (1) early therapy initiation, given that the therapeutic window is likely the 2-3 first weeks following disease onset, (2) a combination of steroidal and first-line non-steroidal immunosuppression, and (3) zero tolerance to subclinical choroiditis, best monitored by ICGA with the need to re-increase therapy if HDDs are still present or reappear.

The disease will evolve toward chronic VKH in the absence of such a strict approach within an appropriate time period, a disease differentiated from initial-onset $\mathrm{VKH}$ and more resistant to therapy, not prone to remission, and often leading to ocular (SGF, subretinal fibrosis, glaucoma) and extra-ocular complications including integumentary changes (vitiligo, alopecia, poliosis) and hearing loss.

\section{Compliance with ethical standards}

Conflict of interest The authors declare that they have no conflict of interest.

Ethical standards All procedures performed in studies involving human participants were in accordance with the 1964 Helsinki declaration and its later amendments or comparable ethical standards.

Open Access This article is distributed under the terms of the Creative Commons Attribution 4.0 International License (http:// creativecommons.org/licenses/by/4.0/), which permits unrestricted use, distribution, and reproduction in any medium, provided you give appropriate credit to the original author(s) and the source, provide a link to the Creative Commons license, and indicate if changes were made. 


\section{References}

1. Emery P, Seto Y (2003) Role of biologics in early arthritis. Clin Exp Rheumatol 21(Suppl 31):S191-S194

2. Nell VP, Machold KP, Eberl G, Stamm TA, Uffmann M, Smolen JS (2004) Benefit of very early referral and very early therapy with disease-modifying anti-rheumatic drugs in patients with early rheumatoid arthritis. Rheumatology 43:906-914

3. Finckh A, Liang MH, van Herckenrode CM, de Pablo P (2006) Long-term impact of early treatment on radiographic progression in rheumatoid arthritis: a meta-analysis. Arthritis Rheum 55:864-872

4. van Nies JA, Krabben A, Schoones JW, Huizinga TW, Kloppenburg M, van der Helm-van Mil AH (2014) What is the evidence for the presence of a therapeutic window of opportunity in rheumatoid arthritis? A systematic literature review. Ann Rheum Dis 73:861-870

5. Gocho K, Kondo I, Yamaki K (2001) Identification of autoreactive $\mathrm{T}$ cells in Vogt-Koyanagi-Harada disease. Invest Ophthalmol 42:2004-2009

6. Damico FM, Cunha-Neto E, Goldberg AC, Iwai LK, Hammer J, Kalil J, Yamamoto JH (2005) Vogt T-cell recognition and cytokine profile induced by melanocyte epitopes in patients with HLA-DRB1*0405-positive and negative Vogt-Koyanagi-Harada uveitis. Invest Ophthalmol Vis Sci 46:2465-2471

7. Sugita S, Takase H, Taguchi C, Imai Y, Kamoi K, Kawaguchi T, Sugamoto Y, Futagami Y, Itoh K, Mochizukil M (2006) Ocular infiltrating CD4+ T cells from patients with Vogt-Koyanagi-Harada disease recognize human melanocyte antigens. Invest Ophthalmol Vis Sci 47:2547-2554

8. Abu El-Asrar AM, Struyf S, Kangave D et al (2011) Cytokine profiles in aqueous humor of patients with different clinical entities of endogenous uveitis. Clin Immunol 139:177-184

9. Bouchenaki N, Herbort CP (2004) Stromal choroiditis. In: Pleyer U, Mondino B (eds) Essentials in ophthalmology: uveitis and immunological disorders. Springer, Berlin, pp 234-253

10. Fang W, Yang P (2008) Vogt-Koyanagi-Harada syndrome. Curr Eye Res 33:517-523

11. Yang P, Ren Y, Li B, Fang W, Meng Q, Kijlstra A (2007) Clinical characteristics of Vogt-Koyanagi-Harada syndrome in Chinese patients. Ophthalmology 114:606-614

12. Abu El-Asrar AM, Al Tamimi M, Hemachandran S, AlMezaine HS, Al-Muammar A, Kangave D (2013) Prognostic factors for clinical outcomes in patients with VogtKoyanagi-Harada disease treated with high-dose corticosteroids. Acta Ophthalmol 91:e486-e493

13. Mantovaqni A, Resta A, Herbort CP, Abu El Asrar A, Kawaguchi T, Mochizuki M, Okada AA, Rao N (2007) Work-up, diagnosis and management of acute Vogt-Koyanagi-Harada disease: a case of acute myopization with granulomatous uveitis. Int Ophthalmol 27:105-115

14. Iwahashi C, Okuno K, Hashida N, Nakai K, Ohguro N, Nishida K (2015) Incidence and clinical features of recurrent Vogt-Koyanagi-Harada disease in Japanese individuals. Jpn J Ophthalmol 59:157-163
15. Moorthy RS, Inomata H, Rao NA (1995) Vogt-KoyanagiHarada syndrome. Surv Ophthalmol 39:265-292

16. Tugal-Tutkun I, Ozyazgan Y, Akova Y, Sullu Y, Akyol N, Soylu M, Kazoglu H (2007) The spectrum of Vogt-Koyanagi-Harada disease in Turkey: VKH in Turkey. Int Ophthalmol 27:117-123

17. Bouchenaki N, Morisod L, Herbort CP (2000) Vogt-Koyanagi-Harada disease: importance of rapid diagnosis and therapeutic intervention. Klin Monbl Augenheilkd 216:290-294

18. Sakata VM, da Silva FT, Hirata CE, Marin ML, Rodrigues H, Kalil J, Costa RA, Yamamoto JH (2015) High-rate of clinical recurrence in patients with Vogt-Koyanagi-Harada disease treated with early high-dose corticosteroids. Graefes Arch Clin Exp Ophthalmol 253:785-790

19. Chee SP, Jap A, Bascal K (2007) Spectrum of Vogt-Koyanagi-Harada disease in Singapore. Int Ophthalmol 27:137-142

20. Keino H, Goto H, Usui M (2002) Sunset glow fundus in Vogt-Koyanagi-Harada disease with or without chronic ocular inflammation. Graefe's Arch Clin Exp Ophthalmol 240:878-882

21. Abu El-Asrar AM, Al Mudhaiyan T, Al Najashi AA, Hemachandran S, Hariz R, Mousa A, Al-Muammar A (2017) Chronic recurrent Vogt-Koyanagi-Harada disease and development of "sunset glow fundus" predict a worse retinal sensitivity. Ocul Immunol Inflamm 25(4):475-485. https://doi.org/10.3109/09273948.2016.1139730

22. Keino H, Goto H, Mori H, Iwasaki T, Usui M (2006) Association between severity of inflammation in CNS and development of sunset glow fundus in Vogt-KoyanagiHarada disease. Am J Ophthalmol 141:1140-1142

23. Lai TY, Chan RP, Chan CK, Lam DS (2009) Effects of the duration of initial oral corticosteroid treatment on the recurrence of inflammation in Vogt-Koyanagi-Harada disease. Eye 23:542-548

24. Abu El-Asrar AM, Hemachandran S, Al-Mezaine HS, Kangave D, Al-Muammar AM (2012) The outcomes of mycophenolate mofetil therapy combined with systemic corticosteroids in acute uveitis associated with Vogt-Koyanagi-Harada disease. Acta Ophthalmol 90:e603-e608

25. Paredes I, Ahmed M, Foster CS (2006) Immunomodulatory therapy for Vogt-Koyanagi-Harada patients as first-line therapy. Ocul Immunol Inflamm 14:87-90

26. El-Asrar Abu, Dosari M, Hemachandran S, Gikandi PW, Al-Muammar A (2017) Mycophenolate mofetil combined with systemic corticosteroids prevents progression to chronic recurrent inflammation and development of 'sunset glow fundus' in initial-onset acute uveitis associated with Vogt-Koyanagi-Harada disease. Acta Ophthalmol 95:85-90

27. Couto C, Schlaen A, Frick M, Khoury M, Lopez M, Hurtado E, Goldstein D (2016) Adalimumab treatment in patients with Vogt-Koyanagi-Harada disease. Ocul Immunol Inflamm 24:1-5

28. Da Silva FT, Hirata CE, Sakata VM, Olivalves E, Preti R, Pimentel SL, Gomes A, Takahashi WY, Costa RA, Yamamoto JH (2012) Indocyanine green angiography findings in patients with long-standing Vogt-Koyanagi-Harada disease: a cross-sectional study. BMC Ophthalmol 13(12):40. https://doi.org/10.1186/1471-2415-12-40 
29. Bouchenaki N, Herbort CP (2011) Indocyanine green angiography guided management of Vogt-Koyanagi-Harada disease. J Ophthalmic Vis Res 6:241-248

30. Bascal K, Wen DS, Chee SP (2008) Concomitant choroidal inflammation during anterior segment recurrence in VogtKoyanagi-Harada disease. Am J Ophthalmol 145:480-486

31. Miyanaga M, Kawaguchi T, Shimizu K, Miyata Michizuki M (2007) Influence of early cerebrospinal fluid-guided diagnosis and early high-dose corticosteroid therapy on ocular outcomes of Vogt-Koyanagi-Harada disease. Int Ophthalmol 27:183-188

32. Kawaguchi T, Horie S, Bouchenaki N, Ohno-Matsui K, Mochizuki M, Herbort CP (2010) Suboptimal therapy controls clinically apparent disease but not subclinical progression of Vogt-Koyanagi-Harada disease. Int Ophthalmol 30:41-50

33. Damico FM, Bezzera FT, Silva GC, Gasparin F, Yamamoto JH (2009) New insights into Vogt-Koyanagi-Harada disease. Arq Bras Oftalmol 72:413-420

34. Agarwal M, Ganesh SK, Biswas J (2006) Triple agent immunosuppressive therapy in Vogt-Koyanagi-Harada syndrome. Ocul Immunol Inflamm 14:333-339

35. Al-Kharashi AS, Aldibhi H, Al-Fraykh H, Kangave D, Abu El-Asrar AM (2007) Prognostic factors in Vogt-KoyanagiHarada disease. Int Ophthalmol 27:201-210

36. Knecht PB, Papadia M, Herbort CP (2014) Early and sustained treatment modifies the phenotype of birdshot retinochoroiditis. Int Ophthalmol 34(3):563-574

37. Urzua CA, Guerrero J, Gatica H, Velasquez V, Goecke A (2017) Evaluation of glucocortcoid receptor as a biomarker of treatment response in Vogt-Koyanagi-Harada disease. Invest Ophthalmol Vis Sci 58:974-980

38. Herbort CP, Mantovani A, Bouchenaki N (2007) Indocyanine green angiography in Vogt-Koyanagi-Harada disease: angiographic signs and utility in patient follow-up. Int Ophthalmol 27:173-182

39. Miyanaga M, Kawaguchi T, Miyata K, Horie S, Mochizuki M, Herbort CP (2010) Indocyanine green angiography findings in initial acute pre-treatment Vogt-KoyanagiHarada disease in Japanese patients. Jpn J Ophthalmol 54:377-382

40. Abouammoh MA, Gupta V, Hemachandran S, Herbort CP, Abu El-Asrar AM (2016) Indocyanine green angiography in initial-onset acute Vogt-Koyanagi-Harada disease. Acta Ophthalmol. https://doi.org/10.1111/aos.12974

41. Suzuki S (1999) Quantitative evaluation of sunset glow fundus in Vogt-Koyanagi-Harada disease. Jpn J Ophthalmol 43:327-333

42. Chee SP, Jap A (2013) The outcomes of indocyanine green angiography monitored immunotherapy in Vogt-Koyanagi-Harada disease. Br J Ophthalmol 97:130-133

43. Balci O, Gasc A, Jeanin B, Herbort CP Jr (2017) Enhanced depth imaging is less suited than indocyanine green angiography for close monitoring of primary choroiditis: a pilot study. Int Ophthalmol 37:737-748

44. Rubsamen PE, Gass JDM (1991) Vogt-Koyanagi-Harada syndrome: clinical course, therapy, and complications. Arch Ophthalmol 109:682-687

45. Herbort CP Jr, Abu El Asrar AM, Yamamoto JH, Pavésio CE, Gupta V, Khairallah M, Tugal-Tutkun I, Soheilian M, Takeuchi M, Papadia M (2017) Reappraisal of the management of Vogt-Koyanagi-Harada disease: sunset glow fundus is no more a fatality. Int Ophthalmol 37:1383-1395

46. Nakayama M, Keino H, Watanabe T, Okada AA (2018) Clinical features and visual outcomes of 11 patients with new-onset acute Vogt-Koyanagi-Harada disease treated with pulse intravenous corticosteroids. Br J Ophthalmol. https://doi.org/10.1136/bjophthalmol-2017-311691 (Epub ahead of print 18th April 2018) 